\title{
Resolved stellar populations at the distance of Virgo
}

\author{
Eline Tolstoy \\ Kapteyn Astronomical Institute, University of Groningen, \\ Postbus 800, 9700AV Groningen, the Netherlands \\ email: etolstoy@astro.rug.nl
}

\begin{abstract}
Top of the wish list of any astronomer who wants to understand galaxy formation and evolution is to resolve the stellar populations of a sample of giant elliptical galaxies: to take spectra of the stars and make Colour-Magnitude Diagrams going down to the oldest main sequence turn-offs. It is only by measuring the relative numbers of stars on Main Sequence Turnoffs at ages ranging back to the time of the earliest star formation in the Universe that we can obtain unambiguous star formation histories. Understanding star formation histories of individual galaxies underpins all our theories of galaxy formation and evolution. To date we only have detailed star formation histories for the nearest objects in the Local Group, namely galaxies within $700 \mathrm{kpc}$ of our own. This means predominantly small diffuse dwarf galaxies in a poor group environment. To sample the full range of galaxy types and to consider galaxies in a high density environment (where much mass in the Universe resides) we need to be able to resolve stars at the distance of the Virgo $(\sim 17 \mathrm{Mpc})$ or Fornax $(\sim 18 \mathrm{Mpc})$ clusters. This ambitious goal requires an Extremely Large Telescope (ELT), with a diameter of $50-150 \mathrm{~m}$, operating in the optical/near-IR at its diffraction limit.
\end{abstract}

\section{Understanding galaxy formation}

To understand the formation of any galaxy we have to investigate its stellar components, which carry a memory of the entire star forming history of a galaxy. We know that galaxies fall more or less into the Hubble Sequence, but we don't really understand the details of why and how massive galaxies find themselves separated into these different classes. Ellipticals are perhaps the biggest mystery because we don't have an example that is easy to study in our backyard. The Milky Way is a prime example of a "typical" spiral or disc galaxy. It consists of a flattened disc $\left(M_{\text {gas }} \sim 10^{10} M_{\odot} ; M_{\text {disc }} \sim 6 \times 10^{10} M_{\odot}\right)$, with a central bulge $\left(M_{\text {bulge }} \sim 2 \times 10^{10} M_{\odot}\right)$ and a diffuse halo $\left(M_{\text {halo }} \sim 3 \times 10^{9} M_{\odot}\right)$. We also have a second example of a spiral galaxy in the Local Group, namely M31. The stellar content of our Milky Way and M31 appear markedly different. For example, the stellar population in the halo of M31 is more metal rich in the mean than our halo. However, globally both these galaxies contain a large gaseous star-forming disc, a bulge and a halo and both galaxies have significant populations of globular clusters.

It is difficult to reach definitive conclusions on the general formation path of massive galaxies with a sample of just two. However, to enlarge our sample we need to move considerably further away in distance, the Sculptor Group and the M81 group (both at about 2-3 Mpc distance) contain several more large spiral galaxies, but still no elliptical galaxy. For the nearest large elliptical galaxy we have to go to NGC 5128 (Centaurus A, which is a very peculiar galaxy, $\mathrm{S} 0+\mathrm{Spec}$ ) at $3.5 \mathrm{Mpc}$ distance, but unfortunately as this system is very complex it is unlikely to be representative. The Leo Group at $\sim 10 \mathrm{Mpc}$ distance contains the nearest nearly normal elliptical galaxy, NGC 3379 (E1 or S0). But 
of course the Virgo cluster is the real prize for studying elliptical galaxies. Virgo at an average distance of $17 \mathrm{Mpc}$, with over 2000 member galaxies of all morphological types, is the nearest large cluster of galaxies. It has a sequence of bright elliptical galaxies that was already cataloged by Messier in the 18th century, and a luminosity sequence reaching down the smallest dwarf elliptical (e.g., Gavazzi et al. 2005), such as NGC 4486B, believed to be an analog of the Local Group compact dwarf elliptical, M32. It is only in the Virgo cluster that we can truly sample the population variations of elliptical galaxies.

Studying galaxies in Virgo also allows us to sample all types of galaxy in a dense region of the Local Universe (e.g., Hudson 1993), where we believe that the majority of galaxies in the Universe form and evolve. This will allow us to study the differences in the evolutionary progress of galaxies in dense versus diffuse environments.

If we can't observe resolved stars in Virgo we are neither sampling the stellar population of elliptical galaxies nor the high density regions of the Universe.

\section{Resolving individual stars in elliptical galaxies}

It has been shown many times in the past that it is not possible to determine accurate star formation histories going back to the oldest times without resolving stars on the oldest main sequence turnoffs $\left(\mathrm{M}_{V}=+2\right.$ to +4$)$. This is because the same age-metallicity degeneracy which plagues accurate interpretation of integrated spectra is equally a problem for the accurate interpretation of the age and metallicity variation of a stellar population based solely upon photometry of stars on its red giant branch (e.g. Tolstoy 1998; Aparicio \& Gallart 2004).

At the present time it is not possible to detect individual resolved stars on the red giant branch in galaxies at distances greater than $\sim 10 \mathrm{Mpc}$ (with HST), and the oldest main sequence turnoff stars $\left(\mathrm{M}_{V}=+4\right)$ can only be detected out to about $700 \mathrm{kpc}$ distance (also using HST). Hence all studies of large galaxies, such as ellipticals in dense environments (such as large clusters) have been made using the integrated colours and spectra. This only gives limits on the average age of the stellar populations and cannot provide an accurate, detailed star formation history. Of course it is the best tool we have, and it may work quite effectively if carefully applied, but we have no way of testing it at present. There are considerable on-going efforts to glean information about elliptical galaxy properties from integrated spectroscopy, their spectra typically resemble that of a metal rich (solar or higher) K-giant star, and so there are likely to have been very few, if any stars formed in the last 1-2 Gyr. There are tight correlations between indicators of age and metallicity that suggest a limited range of both (e.g., Jørgensen 1997). But the age-metallicity degeneracy make firm conclusions highly uncertain, and model dependent (e.g., Worthey 1993).

Despite considerable efforts elliptical galaxies remain largely mysterious, although some progress has been made. Commonly accepted wisdom used to be that elliptical galaxies are simple systems: gas-free, disc-free, rotationally flattened ellipsoids containing only very old stars. As people have looked more carefully most of these assumptions turn out to be wrong or at best crude approximations. Massive elliptical galaxies are not flattened by rotation, but are anisotropic; they do have an interstellar medium, but it is a very hot medium $\left(\mathrm{T}>10^{6} \mathrm{~K}\right)$ that is difficult to detect except in X-rays. A significant fraction of elliptical galaxies contain peculiar kinematic components (such as counterrotating cores) which suggest a violent process of formation through merging of two or more systems over their history. Furthermore, all ellipticals (and spiral bulges) seem to contain super-massive black holes amounting to $\sim 0.2 \%$ of their mass. It has also been seen that on careful examination elliptical galaxies frequently contain faint stellar discs, 
and low mass ellipticals sometimes appear to contain intermediate age stars. But to understand properly and quantify these general inferences from presently available data we need to resolve individuals stars in elliptical galaxies. As most elliptical galaxies are predominantly old we need to observe individual stars on main sequence turnoffs down to the oldest ages (i.e., $\mathrm{M}_{V} \sim+4$ ).

In Virgo there is a large range of elliptical galaxies to chose from, both in type (dwarf, giant or S0) and luminosity. For example, M87 is one of the brightest elliptical galaxies in Virgo, it lies right in the centre of the gravitational potential and X-ray emission of the cluster. M59 is another luminous elliptical which is considerably less luminous than M87, but still one of the brightest elliptical galaxies in Virgo. M59 contains around 2000 globular clusters, easily 10 times more than the Milky Way. It has a central surface brightness, $\mathrm{I}_{0}=15.8 \mathrm{mag} \operatorname{arcsec}^{-2}$ (fairly typical for the giant elliptical galaxies in Virgo). M59 is one of the largest elliptical galaxies with a half-light radius, $\mathrm{R}_{e}=39^{\prime \prime}(3.25 \mathrm{kpc})$. NGC 4486B, a dwarf elliptical, on the other hand has an $\mathrm{R}_{e}=3^{\prime \prime}(0.25 \mathrm{kpc})$, and Virgo contains the complete range in between. The surface brightness at $\mathrm{R}_{e}$ for the giant elliptical galaxies are typically $\mathrm{I}(\mathrm{e}) \sim 20-23 \mathrm{mag} \operatorname{arcsec}^{-2}$, and for dwarf elliptical galaxies this is a very large spread, with M32 type objects such as NGC 4486B with I(e) 16.8mag $\operatorname{arcsec}^{-2}$, but the more diffuse dwarfs have $\mathrm{I}(\mathrm{e})>23-24 \mathrm{mag} \operatorname{arcsec}^{-2}$. The effective radius, $\mathrm{R}_{e}$, varies from $63^{\prime \prime}$ for $\mathrm{M} 87$ to $<5^{\prime \prime}$ for galaxies such as NGC $4486 \mathrm{~B}$, i.e. from the more than $5 \mathrm{kpc}$ to less than 500pc. Thus an optimum field of view for an instrument studying these galaxies should ideally be larger than $2-3^{\prime \prime}$ but need not be larger than about $1^{\prime}$.

These surface brightnesses and angular sizes provide the requirements for both resolution and sensitivity to make accurate colour-magnitude diagrams of resolved stellar populations in elliptical galaxies at the distance of Virgo. If we cannot observe resolved stars in Virgo we will not obtain a detailed understanding of elliptical galaxies, and we will only get an accurate detailed understanding of elliptical galaxies with complete star formation histories coming from Main Sequence Turn-off photometry of individual stars.

\section{Requirements: limiting magnitude \& spatial resolution}

As established in the previous section the requirements for making accurate colourmagnitude diagrams at the distance of Virgo are a combination of increasing spatial resolution with increasing sensitivity.

From deep HST observations of Leo A (Tolstoy et al. 1998), we can show that with the 0.1 arcsec pixels of the Wide Field Camera chip on WFPC2 we were easily able to carry out accurate photometry of stars down the level of the Horizontal Branch $\left(\mathrm{m}_{V}=25\right)$ for the centre of this galaxy with a surface brightness of $\Sigma_{V}=24.5 \mathrm{mag} \operatorname{arcsec}^{-2}$. This resulted in $\sim 2500$ stars per $1 \times 1$ arcmin WF chip $\left(200 \times 200 \mathrm{pc}^{2}\right.$ at the distance of Leo A), or a stellar density of 1 star per $1.44 \operatorname{arcsec}^{2}$. This is by no means crowded. At a distance of Virgo this same surface area is $2.5 \times 2.5 \operatorname{arcsec}^{2}$. Which provides us with a minimum required resolution of $<0.05$ arcsec to carry out the same observation of the same type of galaxy. Obviously elliptical galaxies have typically a considerably higher surface brightness, and hence stellar density, than faint dwarf irregular galaxies. Using for example the surface brightness of an elliptical galaxy in Virgo (e.g., M59, I(e) 22 mag $\operatorname{arcsec}^{2}$, and in the centre, $\left.\mathrm{I}(0) \sim 16 \mathrm{mag} \operatorname{arcsec}^{2}\right)$, we can determine the difficulties in resolving the individual stars, even assuming that we have the sensitivity to detect them. At $\mathrm{R}_{e}$ we would expect an average stellar separation of $\sim 15$ mas between stars 


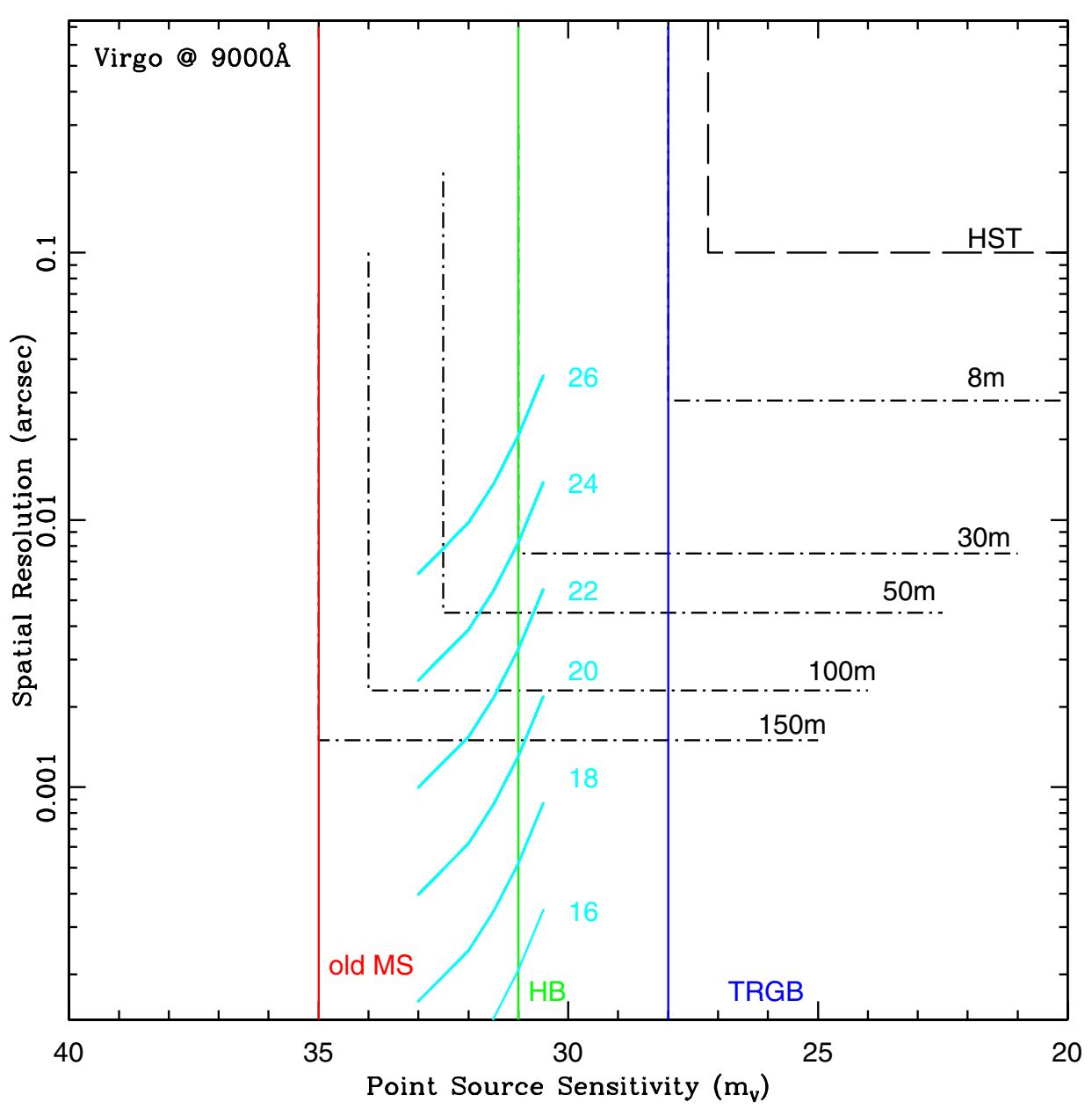

Figure 1. The dashed half-box lines show the parameter space accessible to different aperture telescopes at an observing wavelength of $9000 \AA$. The spatial resolution is simply defined by the diffraction limit of the telescope aperture. The point source sensitivity is determined using the ESO-ELT exposure time calculator (etc.), and assuming a reasonable exposure time (2-4 hours) and a moderate Strehl $(30 \%)$. As a "reality check" we calculated the same limits from the ELT etc. for an 8m telescope. We have also plotted the known limit of HST/WFPC2 from experience observing resolved stellar populations in the Local Group. The 3 vertical lines at $\mathrm{m}_{I}=35,31 \& 28$ represent the approximate observed magnitude, at the distance of Virgo, of the oldest, 13 Gyr, main sequence turnoffs (old MS); the Horizontal Branch (HB) and the tip of the Red Giant Branch (TRGB), respectively. Also plotted are a series of sloping lines centres at $\mathrm{m}_{V} \sim 31.5$ which represent the requirement to resolve the stars in an elliptical galaxy for the point source sensitivity at a variety of surface brightness levels.

at the Horizontal Branch (or 5000 stars per $\operatorname{arcsec}^{2}$ ), and in the centre, $\sim 1$ mas average stellar separation (1 million stars per $\operatorname{arcsec}^{2}$ ), at the Horizontal Branch.

In Fig. 1 we have attempted to visualize these limits in a plot of point source sensitivity versus spatial resolution making an attempt to correct for variations in stellar population. The above numbers assume a constant star formation rate until the present day, but for 
an Elliptical galaxy it is more realistic to assume that star formation stopped at least 5 Gyr ago. The series of sloping lines which represent the requirement to resolve the stars in an elliptical galaxy for a given point-source sensitivity at a variety of surface brightness levels are determined using theoretical stellar evolution models combined to create an artificial stellar population, which is only used in this context to determine the number of stars expected at magnitudes brighter than the point-source sensitivity limit. All the model lines assume the same star formation history (all stars $>5$ Gyr old, and solar metallicity). As the point source sensitivity increases a larger number of stars are detected which decreases the spatial resolution requirement. The associated number is the surface brightness $\left(\Sigma_{V}\right)$ of the "galaxy" (or region thereof) being "observed". It is clear that no telescope so far imagined will be able to resolve stars at the level of the Horizontal Branch, let alone old main sequence turnoffs, in the central regions of Virgo elliptical galaxies. However, with a 50m diffraction limited telescope, with some effort, or better still a $100 \mathrm{~m}$ telescope we can hope to detect and resolve horizontal branch stars in Virgo elliptical galaxies at their effective radius at $9000 \AA$. These are extremely preliminary results which rest on a large number of highly uncertain parameters. It is clear this calculation should be carried out carefully for each different telescope and instrument design. The next logical step is to output the star formation histories here used merely to determine the number of stars in a given area to create an image with realistic point spread functions.

\section{Spectroscopy}

Stars retain a sample of the interstellar medium out of which they were formed in their outer atmosphere; a gaseous envelope which surrounds the nuclear burning core. This envelope is believed to remain unaltered through time for low mass stars, and thus a "message in a bottle" is passed to us giving detailed information about the interstellar medium out of which this star was formed. Many of these stars have very long lifetimes, and some of those we see today will have formed (relatively) soon after the Big Bang. The central core of the star provides a background light source shining though this envelope and the absorption lines we see tell us about the chemical composition of this envelope. The more lines we can observe the more elements we trace the better we understand the message the star is providing for us. Different chemical elements tell us how this interstellar medium was enriched (if at all!) by previous generations of stars in the period before this star was formed. For example, the $\alpha$-elements (O, Ca, Mg etc) can tell us how many and what mass of supernovae type II explosions polluted the interstellar medium before this star was made. Other elements can tell us about the likely contribution of supernovae type I and even stellar winds coming from AGB stars (e.g., McWilliam 1997).

For the most detailed abundance analyses (e.g., Shetrone et al. 2003) high resolution spectroscopy is required $(\mathrm{R} \sim 40000)$ with a reasonably high signal-to-noise $(\sim 40)$. With an optimistic outlook this may be possible for red giant branch stars at the distance of M31, and maybe even Cen A with an ELT type telescope. This would allow us to understand the detailed differences between the stellar populations of M31 and our Milky Way as well as other galaxies in the Local Group (e.g., Venn et al. 2004).

However, if you are able to detect old main sequence stars in a Colour-Magnitude Diagram ( $>2$ Gyr old) you are also able to determine metallicities for individual red giant branch stars from particular strong sets of lines (e.g., the Ca II triplet at $\lambda \lambda=8498$, 8542, $8662 \AA)$ observed at intermediate resolution ( $\mathrm{R} \geqslant$ few thousand), with moderate signal-to-noise $(\geqslant 10)$. The Ca II triplet, for example, has been well calibrated to high resolution metallicity scale (e.g., Cole et al. 2004; Battaglia et al. 2006, in prep.), and 
accurately measure the $[\mathrm{Fe} / \mathrm{H}]$ of a star (not $[\mathrm{Ca} / \mathrm{Fe}] !)$. Although this indicator (and others) don't (yet) provide much detailed information on any element other than Fe they still provide a valuable insight into the chemical evolution of the galaxy which can provide vital information to correctly interpret a star formation history, especially if deep main sequence photometry is not possible.

Ca II triplet metallicities are only meaningful in a statistical sense, because they are individually representative of $[\mathrm{Fe} / \mathrm{H}]$ at a particular instant in the history of the galaxy that is always changing, and so large samples of stars should be observed per galaxy to build up an accurate picture of this variation over the entire star forming history of the galaxy. This requires multi-object spectroscopy over the same field of view as the imaging discussed in the previous section. Imaging is of course also required to make finding charts. The tip of the red giant branch is $\mathrm{M}_{I}=-4$; which at Virgo is $\mathrm{m}(I)=27$. This might be feasible with a $100 \mathrm{~m}$ telescope, but it will most likely be overly challenging for smaller aperture sizes.

\section{Acknowledgements}

The author would like to gratefully acknowledge support from a fellowship of the Royal Netherlands Academy of Arts and Sciences, and travel support from the Leids Kerkhoven Bosscha Fonds. Thanks to Mike Irwin for critical comments and corrections.

\section{References}

Aparicio, A. \& Gallart C. 2004, AJ 128, 1465

Cole, A.A., Smecker-Hane, T.A., Tolstoy, E., Bosler, T.L. \& Gallagher, J.S. 2004, MNRAS 347, 367

Gavazzi, M.J., Donati, A., Cucciati, O., Sabatini, S., Boselli, A., Davies, J. \& Zibetti, S. 2005, A\&SA 430, 411

Hudson, I. 1993, MNRAS 265, 43

Jørgensen, I. 1997, MNRAS 288, 161

McWilliam, A. 1997, ARAA 35, 503

Shetrone, M.D., Venn, K.A., Tolstoy, E., Primas, F., Hill, V. \& Kaufer A. 2003, AJ 125, 684

Tolstoy, E., Gallagher, J.S., Cole, A.A., Hoessel, J.G., Saha, A., Dohm-Palmer, R.C., Skillman, E.D., Mateo, M., Hurley-Keller, D. 1998, AJ, 116, 1244

Tolstoy, E. 1998, in: T.X. Thuan et al. (eds.), Dwarf Galaxies \& Cosmology, p. 171

Venn, K.A., Irwin, M., Shetrone, M.D., Tout, C.A., Hill, V. \& Tolstoy, E. 2004, AJ 128, 1177

Worthey, G. 1993, PASP 105, 326

\section{Discussion}

ZIJLSTRA: Have you considered using planetary nebulae as tracers of abundances and velocities? They are much brighter and representative of older stars.

Tolstoy: They are difficult to fit into an evolutionary context so although abundances can be measured, fitting the measurement into the star formation history is currently not possible.

ARDEBERG: It is easy to agree on the importance of turn-off point photometry for adequate conclusions on evolution. Then, though, the need for spatial resolution, especially in more crowded regions, forces us to the shorter wavelength region. How do you see the trade-off between high spatial resolution and easier NIR access in terms of AO PSFs?

Tolstoy: The environment of field star populations in the outer region of elliptical galaxies (for example) is such that it is possible to chose the degree of crowding by being 
flexible about placement of the field of view. Of course care has to be taken that sufficient stars are detected in the available field of view - the trade off between resolution crowding and field size need to be carefully considered in instrument (and telescope) design.

CRAmpton: To reach Virgo we will need to go to the NIR. Do you have confidence that we can determine accurate, reliable abundances from NIR photometry and spectroscopy in the NIR?

Getting down to $\lambda \sim 0.7 \mu \mathrm{m}$ with $\mathrm{AO}$ is feasible in my opinion: the Strehl decreases dramatically, but the FWHM is relatively constant from $K$ to $R$, and below $1 \mu \mathrm{m}$ the wings shrink as well. (Rigaut et al. 1996?)

Tolstoy: I think the field of NIR Spectroscopy abundances of old stars is very young, but there are claims that it can provide useful abundance information. 